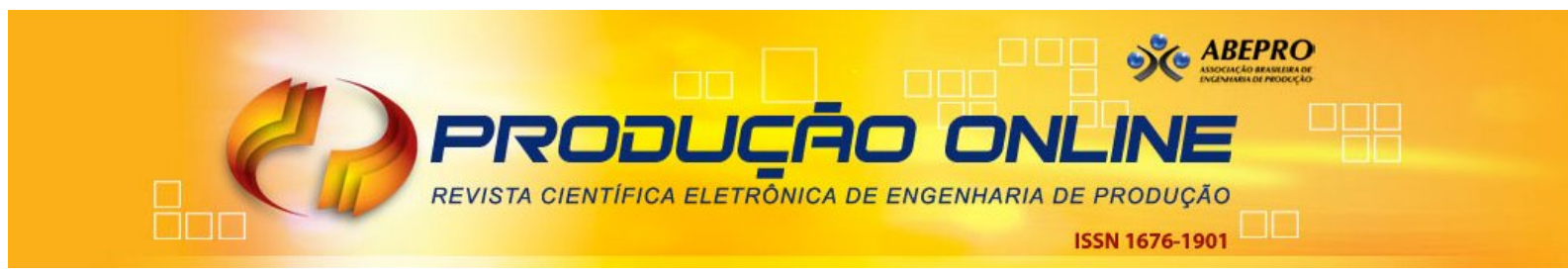

\title{
INTEGRAÇÃO DO SEIS SIGMA COM O LEAN PRODUCTION: UMA ANÁLISE POR MEIO DE MÚLTIPLOS CASOS
}

\section{INTEGRATION OF SIX SIGMA WITH LEAN PRODUCTION: AN ANALYSIS THROUGH MULTIPLE CASES}

\author{
Thiago Henrique Pinheiro`E-mail: thiago.pinheiro@poli.usp.br \\ Alisson Christian Scheller**E-mail: alisson.christianscheller@gmail.com \\ Paulo A Cauchick Miguel** E-mail: cauchick@deps.ufsc.br \\ *Universidade de São Paulo (USP), São Paulo, SP \\ **Universidade Federal de Santa Catarina (UFSC), Florianópolis, SC
}

\begin{abstract}
Resumo: A forte concorrência entre os mercados tem levado as organizações, independentemente do porte e setor industrial, a implementarem um ou mais programas de melhoria da qualidade. Um dos propósitos destes programas é a melhoria da eficácia e eficiência organizacional para quem os implementa. Uma proposta denominada Lean Six Sigma tem despertado interesse, dado que seu objetivo é aplicar os conceitos do programa Seis Sigma integrando-os aos princípios do Lean Production. O Seis Sigma contribui com meios de identificação, medição e análise do problema, enquanto que o Lean Production oferece técnicas e procedimentos aplicados para a redução de desperdícios na produção. Nesse contexto, o objetivo desse trabalho é demonstrar os resultados de um trabalho de campo, cujo propósito foi identificar os fatores importantes para a implementação do Seis Sigma combinado com os princípios do Lean Production. Para cumpri-lo, três organizações de diferentes setores industriais foram investigadas. Estas organizações aplicam tanto o Seis Sigma quanto o Lean Production. Constata-se que os programas foram implementados com sucesso em apenas duas das empresas estudadas. Por meio da análise dos dados é possível identificar que uma das maiores dificuldades encontradas pelos líderes dos programas diz respeito à (falta de) infraestrutura de recursos humanos nas organizações. Deve-se levar em conta, no entanto, que as conclusões do presente estudo estão limitadas a pequena amostra investigada, que se restringe a três unidades de análise. Não obstantemente, o trabalho oferece a possibilidade de uma generalização analítica.
\end{abstract}

Palavras-chave: Lean six sigma. Seis sigma. Manufatura enxuta. Gestão da qualidade.

\begin{abstract}
The intense competition between markets has taken companies, regardless of their size, to implement one or more quality improvement programs. One of the main purposes of these programs is the enhancement of company effectiveness and efficiency. There is today a growing interested in a proposal named Lean Six Sigma. Their objective is to apply six sigma concepts integrated with Lean Production. A model named Lean Six Sigma has raised interest, once its objective is to apply the concepts of the Six Sigma program integrating them with the principles of Lean Production. Six Sigma contributes with methods for problem identification, measurement and analysis, and the Lean Production offers techniques and procedures applied to reduce waste in the production. In this context, this paper aims at demonstrating the results of a field research, from which purpose was to identify relevant factors for Six Sigma implementation combined with Lean Production. To meet these objectives, three organizations from different industrial sectors were investigated. It was verified that in two of those companies, the programs were successfully implemented. Based on data analysis, it is possible to identify that one of the major difficulties encountered by the program leaders is related to the company's human resources infra-structure. It is necessary to take into account that the
\end{abstract}


conclusions are limited to a small sample of three units of analysis. However, this work offers the possibility of an analytical generalization.

Keywords: Lean six sigma. Six sigma. Lean production. Quality management.

\section{INTRODUÇÃO}

Já a algum tempo, independentemente do segmento e mercado de atuação, as organizações devem focar em velocidade, eficiência e valor para o cliente para se tornarem competitivas globalmente (CUDNEY; ELROD, 2011). Complementando este argumento, a competitividade no meio industrial cresceu consideravelmente nas últimas décadas e, como consequência direta, a busca por técnicas diferenciadas para a obtenção de melhor desempenho em todos os processos tem sido uma das principais preocupações das organizações interessadas em consolidar sua presença nesse mercado global (FERNANDES; TURRIONI, 2007). É essencial que as organizações estejam adaptadas a atender as necessidades de seus clientes a fim de disponibilizar um ambiente flexível e eficaz (FIORE, 2003). Portanto, para atender às exigências do mercado tem-se como pré-requisito produzir a custos baixos e reduzir a quantidade de defeitos (HARRY; SCHROEDER, 2000). Um processo com elevada variabilidade resulta em produtos ou serviços de baixa qualidade, com altos custos e consequente insatisfação dos clientes. Dessa forma, busca-se sempre produzir produtos uniformes (GEORGE, 2002).

A necessidade de aumento não apenas da qualidade, mas também de produtividade, fazem com que haja a busca pela melhoria contínua (SALAH et al., 2010). Nesse sentido, diversas empresas têm adotado um ou mais programas de qualidade para se tornarem mais competitivas. Nas últimas décadas, surgiram vários programas tais como as normas da série ISO 9000 (International Organizational for Standardization), a gestão pela qualidade total (TQM - Total Quality Management), a manutenção produtiva total (TPM - Total Productive Maintenance), entre outros. O objetivo principal destes programas é o mesmo: a melhoria da eficácia e eficiência das empresas que os implementam. No entanto, há diferenças significativas entre o processo de implementação dos mesmos e o nível de resultados atingidos (PORTER, 2002). Uma proposta denominada Lean Six Sigma tem sido discutida mais recentemente. Seu principal propósito é aplicar os conceitos do programa Seis 
Sigma integrando-os aos princípios do Lean Production. Como o próprio nome sugere, o Lean Six Sigma é um programa que se baseia nos métodos e ferramentas do Lean Production (Produção Enxuta) e também do programa Seis Sigma.

Os resultados obtidos por algumas das organizações que implementaram o programa Lean Six Sigma, têm despertado a atenção em diferentes ramos de negócios, principalmente, pelos resultados financeiros alcançados por estas empresas (GEORGE, 2002). A junção das características destes dois programas possibilita um maior desempenho organizacional, de modo mais condizente com a realidade competitiva atual, na busca de atingir uma maior qualidade e satisfação dos clientes com os produtos e serviços oferecidos no mercado. Assim, quando os princípios presentes nos dois programas se complementam, um grupo de características reforça as outras, permitindo a obtenção de maiores ganhos. O Lean Six Sigma exige modificações em diversos aspectos de uma organização tais como na cultura empresarial e coordenação da força de trabalho, desde os funcionários operacionais da linha de produção até os executivos da alta da administração.

Nesse contexto, o objetivo desse artigo é demonstrar os resultados de um trabalho de campo, cujo propósito foi identificar os fatores importantes para a implementação Seis Sigma combinado com os princípios do Lean Production. Para a consecução destes objetivos, três organizações de diferentes setores industriais foram investigadas. Estas organizações aplicaram ambos os programas e o presente trabalho visa analisar a adoção dos mesmos. Os resultados obtidos por estas organizações, que buscam a excelência operacional por meio do Lean Six Sigma, são então confrontados com a teoria. Em seguida, alguns fatores relevantes na implementação, bem como as dificuldades e benefícios decorrentes da introdução dos programas são verificados. As três empresas investigadas utilizam ou, em algum momento, utilizaram o Seis Sigma e o Lean Production. A partir do referencial teórico apresentado na seqüência, este artigo descreve a abordagem metodológica de pesquisa adotada, seguido pelos resultados decorrentes dos métodos empregados, e por fim as conclusões do estudo e sugestões para sua continuidade. 


\section{REFERENCIAL TEÓRICO SOBRE LEAN SEIS SIGMA}

Dentre as várias metodologias de melhoria contínua, o Lean Production e o Seis Sigma são possivelmente as mais populares e mais comumente usadas por empresas de diversos setores industriais (GEORGE, 2002; SALAH et al., 2010). A ideia de unir estas metodologias não é recente, de acordo com Straatmann (2006) a visão do Lean Production e do Seis Sigma trabalhando em um mesmo processo de melhoria teve seu início na década de 90, quando as empresas começaram a empregá-los em paralelo e de modo desagregado. O emprego das metodologias dessa forma começou a ocasionar, segundo Bossert (2003) e Smith (2003), alguns problemas e dificuldades, visto que as duas metodologias possuem objetivos distintos (apesar de complementares) e formas diferenciadas de implementação. Uma solução seria a utilização dessas metodologias de modo combinado. Sheridan (2000) denominou Lean Sigma o sistema que combina simultaneamente tanto o Lean como o Seis Sigma. Outros autores se referem a esta integração como Six Sigma Lean (e.g. BYRNE et al., 2007), ou Lean Six Sigma (e.g. MADER, 2008; UPTON, COX, 2008 apud SALAH et al., 2010).

A percepção de que o Lean Production e o Seis Sigma têm uma relação complementar é amplamente aceita atualmente e cada vez mais empresas vem aderindo a estas metodologias, especialmente após terem a sua capacidade de gerar resultados demonstrada em empresas líderes tais como a General Electric e a Toyota (SALAH et al., 2010). Existe uma relação óbvia entre o Seis Sigma, que foca na correção de processos individuais, enquanto que o Lean Production centra esforços nas conexões entre os processos (ARTHUR, 2007). Ambas as metodologias dão ênfase no fluxo de processos e operações. Antony (2010) faz uma comparação sobre o foco de cada metodologia. O autor destaca que o Lean Production objetiva a redução de desperdícios, aumento de produtividade e agilidade do fluxo, e eliminação das atividades que não agregam valor a fim de reduzir custos, enquanto que o Seis Sigma tem o propósito da redução da variabilidade e o combate sistemático à produção de itens de baixa qualidade, reduzindo assim os custos. 
O Lean Seis Sigma, segundo Salah et al. (2010), pode ser definido como uma metodologia que foca na eliminação de desperdício e variação nos processos, seguindo a estrutura DMAIC (Define, Measure, Analyze, Improve, Control), a fim de atingir a satisfação dos clientes, com qualidade e custo mais baixo. Como destacado, seu foco é tanto na melhoria de processos quanto na busca de melhor desempenho financeiro para a empresa. Além de todos os benefícios gerados pela junção destas metodologias, relativos ao aumento da qualidade, redução de custo e do lead time e maior satisfação dos clientes, Welch (2005) afirma que talvez o maior benefício do Lean Seis Sigma seja sua capacidade de desenvolver um quadro de grandes líderes.

\subsection{Similaridades entre o Lean Production e o Seis Sigma}

Visando compreender a operação do Lean Seis Sigma, é importante mostrar uma comparação entre o Lean Production e o Seis Sigma. Antony (2010) afirma que, certamente, uma abordagem integrada entre as duas metodologias produz resultados significativos e sustentáveis. O autor citado lista uma série de semelhanças entre a abordagem do Lean Production e do Seis Sigma:

(i) são processos centrais da organização;

(ii) podem ser aplicados em atividades além da manufatura

(iii) precisam de suporte da gestão, especialmente em termos de criar a infraestrutura necessária e mudar a cultura do negócio;

(iv) focam na necessidade do cliente;

(v) utilizam equipes multifuncionais para trabalhar nos problemas da organização; e

(vi) oferecem ferramentas complementares entre si capazes de transformar os processos organizacionais em direção a excelência em desempenho.

Também são destacadas algumas diferenças entre as duas metodologias (ANTONY, 2010):

(i) o Seis Sigma requer treinamento mais intensivo comparado com o Lean Production; 
(ii) a implementação do Seis Sigma requer maior investimento do que a do Lean Production;

(iii) o Lean Production é fundamentalmente utilizado para resolver ineficiências de processos enquanto o Seis Sigma é usado para aumentar a eficácia; e

(iv) o Seis Sigma elimina defeitos nos processos, porém não se atém a questões de como otimizar o fluxo entre processos. Em contraste, os princípios do Lean Production não ajudam tanto em aumentar a capabilidade e diminuir a variabilidade do processo.

Em um estudo mais detalhado, Salah et al. (2010), com base em Yang (2004), fizeram uma revisão sobre o tema e elaboraram uma lista de dimensões comparativas entre o Lean Production e o Seis Sigma. O Quadro 1 apresenta algumas dessas similaridades, de acordo os autores supracitados.

Quadro 1 - Similaridades entre o Seis Sigma e o Lean Production.

\begin{tabular}{|c|c|c|c|}
\hline & Seis Sigma & Lean Production & Observações \\
\hline Origem & Motorola (década de 80) & Toyota (década de 70) & $\begin{array}{l}\text { Ambos incorporam } \\
\text { princípios do TQM } \\
\text { (Total Quality } \\
\text { Management) }\end{array}$ \\
\hline Liderança & $\begin{array}{l}\text { Abordagem de cima para } \\
\text { baixo ("top down") }\end{array}$ & $\begin{array}{l}\text { Abordagem de cima para } \\
\text { baixo ("top down") }\end{array}$ & $\begin{array}{l}\text { Ambos enfatizam } \\
\text { comprometimento e } \\
\text { suporte da liderança } \\
\text { (YANG, 2004) }\end{array}$ \\
\hline $\begin{array}{l}\text { Princípios, } \\
\text { métodos e } \\
\text { ferramentas }\end{array}$ & $\begin{array}{c}\text { Capabilidade e } \\
\text { estabilidade dos } \\
\text { processos, DMAIC, CTQs } \\
\text { (Critical to Quality) (HAN; } \\
\text { LEE, 2002); voz do cliente, } \\
\text { eliminação de defeitos } \\
\text { (YANG, 2004) }\end{array}$ & $\begin{array}{c}\text { Busca de valor, } \\
\text { quantidade e qualidade } \\
\text { para os clientes quando } \\
\text { ele necessita; } 5 \text { princípios } \\
\text { (WOMACK, JONES, 1991) }\end{array}$ & $\begin{array}{l}\text { Ambos têm traços do } \\
\text { TQM, que pode ser } \\
\text { visto como um pilar } \\
\text { do Lean Seis Sigma } \\
\text { (SHEEHY et al., } \\
\text { 2002) }\end{array}$ \\
\hline $\begin{array}{l}\text { Características } \\
\text { de estrutura }\end{array}$ & $\begin{array}{l}\text { Gerenciamento de } \\
\text { projetos com plano de } \\
\text { melhoria }\end{array}$ & $\begin{array}{l}\text { Gerenciamento de } \\
\text { projetos com } \\
\text { implementação de } \\
\text { melhorias pelo MFV }\end{array}$ & $\begin{array}{l}\text { Ambos guiados pela } \\
\text { liderança com base } \\
\text { em planejamento e } \\
\text { execução } \\
\text { (ANDERSON et al., } \\
2006)\end{array}$ \\
\hline Participantes & $\begin{array}{c}\text { Green belts, black belts, } \\
\text { masters black belts e } \\
\text { champions }\end{array}$ & $\begin{array}{l}\text { Líder de Kaizen, Líder do } \\
\text { Mapeamento do Fluxo de } \\
\text { Valor (MFV), Sensei }\end{array}$ & - \\
\hline
\end{tabular}

Fonte: Salah et al. (2010); adaptado pelos autores. 
Como pode ser visto no Quadro 1, existem aspectos em comum entre o Lean Production e o Seis Sigma: ambos tem origem nos princípios da qualidade total (TQM); necessitam de suporte da liderança para introdução e continuidade, sendo introduzidos com uma abordagem de cima para baixo; são orientadas por princípios básicos, métodos e ferramentas, possuem características que objetivam a melhoria contínua e definem funções básicas para as pessoas envolvidas nestas metodologias.

Segundo Salah et al. (2010), a abordagem do Lean Seis Sigma deve levar em conta a diferença entre as metodologias, pois se complementam. Desta forma, a integração é possível e benéfica, como destacado a seguir.

\subsection{Integração entre o Lean Production e o Seis Sigma}

É esperado que o Seis Sigma seja trabalhado em conjunto com outras metodologias de melhoria contínua (ANTONY, 2004). Com relação ao Lean Production, Hines et al. (2004) indicam que também é possível integrá-lo com outras abordagens sem contradizer seus objetivos de entregar valor ao cliente. O Lean Production e o Seis Sigma não devem ser usados em paralelo, mas simultaneamente para que a sinergia entre ambos seja aproveitada e evite dificuldades como priorizar iniciativas e alocar recursos (SALAH et al., 2010). A integração é o caminho para que a organização aumente o seu potencial de melhoria (BHUIYAN; BAGHEL, 2005).

Segundo Smith (2003), esta integração permite alcançar a melhoria contínua, atingir zero defeitos e entrega rápida a baixo custo. A tentativa de trabalho com ambas as metodologias atuando em paralelo nem sempre é bem sucedida, uma vez que as ferramentas para resolução de problemas são aplicadas separadamente (SALAH et al., 2010). Um dos requisitos fundamentais para sucesso nos esforços de melhoria contínua em uma organização é a disponibilidade de um conjunto comum de ferramentas para a resolução de problemas (CHAPMAN; HYLAND, 1997). As ferramentas utilizadas no Lean Production e no Seis Sigma podem ser consideradas como uma caixa de ferramentas, onde, dependendo da natureza do problema e sua origem, é possível escolher a mais apropriada entre elas (MCADAM; DONEGAN, 
2003). Com isso é possível usá-las tanto de forma rápida como em eventos Gemba Kaizen, ou seja, com projetos de melhoria com equipes focadas e duração aproximada de uma semana, como também em análises profundas em projetos mais complexos, com duração de alguns meses. Segundo Antony (2010), esta integração oferece resultados superiores do que quando utilizadas em separado. O mesmo autor afirma que enquanto o Lean Production busca eliminar os desperdícios e atividades que não agregam valor na organização, o Seis Sigma através de técnicas e ferramentas estatísticas, leva a melhoria de performance e capabilidade dos processos.

Salah et al. (2010) listam seis possibilidades de aplicação relacionadas ao Lean Seis Sigma encontrados nas organizações:

i. O Lean Production como metodologia principal e o Seis Sigma como ferramenta complementar em eventos Gemba Kaizen.

ii. O Seis Sigma como metodologia principal e as ferramentas do Lean Production aplicadas dentro da estrutura DMAIC.

iii. O Lean Production e o Seis Sigma em separado para tratar de diferentes problemas de acordo com a classificação do projeto.

iv. O Lean e o Seis Sigma em paralelo para a resolução dos mesmos problemas, porém separadamente.

v. Aplicação do Lean Production e do Seis Sigma continuamente para atacar os mesmo problemas.

vi. Aplicação do Lean Production e do Seis Sigma simultaneamente.

A metodologia Lean Seis Sigma ainda divide opiniões e ainda não existe um modelo conceitual claro e estruturado para sua aplicação, principalmente porque muitas empresas já trabalham com o Lean Production ou com o Seis Sigma, o que dificulta a criação de uma proposta única de implementação. De acordo com Salah et al. (2010), o sucesso da integração destas metodologias depende de as organizações terem uma visão holística de melhoria contínua em que o Lean Production e o Seis Sigma mutuamente reforçam um ao outro. Os mesmos autores também afirmam que apesar de o modelo DMAIC ter sua origem no Seis Sigma, este pode ser generalizado como um modelo global para a melhoria contínua. Um 
projeto que utiliza a metodologia DMAIC deve se referir a um problema de desempenho organizacional, o qual tem uma solução desconhecida (CLETO, QUINTEIRO, 2011). Segundo os autores citados, deve haver um conjunto de objetivos mensuráveis ligados a indicadores bem definidos e que correspondam à oportunidade de solução, dentro de uma perspectiva de melhoria contínua. Montgomery (2010) sugere que apesar de as ferramentas utilizadas diferirem entre as fases, o uso do modelo DMAIC é tão efetivo no Lean Production quanto é no Seis Sigma. Segundo dados colhidos por George (2002), as ações de melhoria que não seguem o DMAIC se dão muito lentamente. O DMAIC propicia uma característica única de conectar e sequenciar as ações de melhoria de uma forma global (SNEE, 2004), de forma a se atingir a excelência operacional (MONTGOMERY, 2010). Salah et al. (2010) recomendam sempre seguir a estrutura DMAIC ao realizar qualquer projeto de Lean Seis Sigma. Para se ter sucesso na aplicação do Seis Sigma é necessário saber exatamente onde, por que, quando e como suas ferramentas são aplicadas no DMAIC (ANTONY, 2004).

\subsection{Fatores de Sucesso para o Lean Seis Sigma}

Jeyaraman e Teo (2010), baseados em estudos feitos por Antony e Banuelas (2002), Spanyi, Wurtzel e PQA (2003 apud JEYARAMAN; TEO, 2010), Waxer (2004 apud JEYARAMAN; TEO, 2010) e Achanga et al. (2006), identificaram uma lista com 25 fatores críticos para o sucesso da implementação do Lean Seis Sigma. Estes fatores representam, segundo os autores, os ingredientes essenciais sem os quais as iniciativas têm poucas chances de sucesso. Os fatores críticos de sucesso não são objetivos, mas sim ações e processos que devem ser controlados para se alcançar as metas organizacionais (BROTHERTON; SHAW, 1996). O estudo desenvolvido pelos autores usou como base os dados enviados por 25 multinacionais que selecionaram os 10 entre os 25 fatores críticos de sucesso que consideravam mais relevantes na implementação do Lean Seis Sigma. Os fatores são listados a seguir, por ordem de importância (JEYARAMAN; TEO, 2010):

i. Compromisso e dedicação da liderança.

ii. Sistema de premiação e reconhecimento. 
iii. Cultura organizacional.

iv. Comunicação frequente e avaliação dos resultados do programa.

v. Priorização, seleção, revisão e acompanhamento de projetos.

vi. Programa efetivo de treinamento.

vii. Compartilhamento de melhores práticas de projetos.

viii. Capacidade financeira da empresa.

ix. Definição do funcionamento do programa e sua ligação com a estratégia da empresa.

x. Competência dos master black belts e black belts.

Pepper e Spedding (2010) citam outros fatores para o sucesso da metodologia, que deve ser: estratégico e focado no processo; balanceado entre as duas metodologias para obter as vantagens de ambos; estruturado de acordo com o tipo problema e balanceado entre a complexidade e sustentabilidade da abordagem. Martin (2007) também indica alguns fatores chave para o sucesso das iniciativas do Lean Seis Sigma como: (i) suporte da liderança; (ii) escolha das pessoas certas para participação; (iii) seleção dos projetos certos para serem conduzidos; (iv) comunicação efetiva e possuir as características de um programa efetivo de mudanças. O Quadro 2 apresenta uma síntese dos fatores críticos de sucesso da implementação do Lean Seis Sigma de acordo com os diferentes autores citados.

O Lean Seis Sigma aparenta ser uma metodologia estabelecida, confirmada por diversos autores (GEORGE, 2002; ARNHEITER; MALEYEFF, 2005; KUMAR et al., 2006; WEDGWOOD, 2006; SALAH et al., 2010), entretanto, de acordo com Chiarini (2011), alguns fatores críticos de sucesso do Lean Production, como o envolvimento de todas as pessoas, não são levados em consideração ou são negligenciados. Segundo o mesmo autor, muitas propostas de integração do Lean Seis Sigma são baseadas na certificação de Black belts e Green belts, porém, essa estrutura se assemelha mais ao Seis Sigma enriquecido com algumas ferramentas e métodos do Lean Production. Infelizmente ainda não existe na literatura ou em treinamentos profissionais, referências específicas de como balancear e enfatizar os fatores intrínsecos que fazem crescer o envolvimento e crescimento das pessoas (CHIARINI, 2011). 
Quadro 2 - Fatores críticos de sucesso de acordo com diferentes autores

\begin{tabular}{|c|c|c|}
\hline $\begin{array}{c}\text { Jeyaraman e Teo (2010) } \\
\text { da liderança }\end{array}$ & $\begin{array}{c}\text { Pepper e Spedding (2010) } \\
\text { processo }\end{array}$ & Martin (2007) \\
\hline $\begin{array}{c}\text { Comproma de premiação e } \\
\text { reconhecimento }\end{array}$ & $\begin{array}{c}\text { Balanceado entre a } \\
\text { complexidade e } \\
\text { sustentabilidade da } \\
\text { abordagem }\end{array}$ & Escolha das pessoas certas \\
\hline $\begin{array}{c}\text { Cultura organizacional } \\
\text { Comunicação frequente e } \\
\text { avaliação dos resultados } \\
\text { do programa }\end{array}$ & $\begin{array}{c}\text { Balanceado entre as duas } \\
\text { metodologias }\end{array}$ & Seleção dos projetos certos \\
\hline $\begin{array}{c}\text { Priorização, seleção, } \\
\text { revisão e } \\
\text { acompanhamento do } \\
\text { programa }\end{array}$ & o tipo de problema & Comunicação efetiva \\
\hline $\begin{array}{c}\text { Programa de treinamento } \\
\text { efetivo }\end{array}$ & - & Características efetivas de \\
mudanças
\end{tabular}

Fonte: tabela construída pelos autores com base nas referências bibliográficas indicadas.

\section{MÉTODOS E TÉCNICAS DE PESQUISA}

Nesta seção, pretende-se fornecer uma visão geral sobre a abordagem metodológica de pesquisa utilizada no presente trabalho, bem como uma justificativa para a seleção dos métodos e técnicas empregados para a coleta e análise dos dados. A importância da abordagem metodológica de um trabalho pode ser justificada pela necessidade de embasamento científico adequado, usualmente buscando-se a melhor abordagem metodológica para se atingir os objetivos de um 
projeto de pesquisa, assim como seus respectivos métodos e técnicas para seu planejamento e condução (CAUCHICK MIGUEL, 2007). O delineamento da pesquisa está relacionado ao processo completo de planejamento e de execução da pesquisa, em que se parte dos objetivos iniciais e se finaliza com a apresentação de resultados conclusivos na forma de um documento padrão (CRESWELL, 1994).

Segundo Fillippini (1997), Fillippini e Voss (1997), Berto e Nakano (2000), e Cauchick Miguel (2007), as principais abordagens de pesquisa utilizadas na engenharia de produção e gestão das operações são: estudo de caso; desenvolvimento teórico-conceitual; levantamento tipo survey; modelagem e simulação; pesquisa-ação; pesquisa bibliográfica; pesquisas experimentais. No presente trabalho, a abordagem metodológica adotada é o estudo de caso, que permite aproximar o pesquisador das circunstâncias que envolvem o fenômeno que se deseja estudar, abordando-o de forma mais ampla, uma vez que se utilizam múltiplas fontes para coleta de dados, sendo recomendado quando o pesquisador tem baixo controle sobre os eventos observados (BRYMAN, 1989; YIN, 2005). O presente trabalho apresenta características relacionadas a estes fatores, justificando assim a escolha da abordagem de estudo de caso. Além disso, esta abordagem de pesquisa deve ser aplicada com o objetivo de se aprofundar o conhecimento de um problema ainda não muito bem definido (MATTAR, 1996), como se apresenta o tema do presente estudo, uma vez que a metodologia Lean Six Sigma ainda não se encontra plenamente consolidada sob o ponto de vista empírico.

Com o intuito de minimizar os erros associados à condução de estudo de casos, o trabalho de pesquisa seguiu algumas recomendações indicadas por Cauchick Miguel (2007) para o planejamento e condução de casos múltiplos. Primeiramente, na fase inicial deste trabalho realizou-se o mapeamento da literatura, organizando a literatura em um banco de dados de artigos científicos publicados em periódicos internacionais arbitrados relevantes e, principalmente, diretamente relacionados ao tema de pesquisa. Desse modo, a ênfase no presente trabalho está nas publicações sobre o tema Lean Six Sigma.

Após essa fase, foi adaptado um questionário existente, cedido pelos pesquisadores (ANDRIETTA; CAUCHICK MIGUEL, 2007), para ser aplicado às empresas visitadas, a fim de caracterizar os programas Seis Sigma e Lean 
Production, bem como examinar como ocorre a integração entre estas metodologias naquelas organizações. Na coleta dos dados de campo, selecionaram-se algumas empresas a partir do critério principal de identificação de organizações que empregam ou em algum momento empregaram os programas concomitantemente. Assim, foram identificadas algumas empresas, de grande porte, pertencentes a ramos diferentes de atividade econômica. Foram contatadas 8 empresas, sendo que 3 aceitaram participar do estudo. Estas empresas possuem destaque no mercado nacional, com ampla participação de mercado nos seus respectivos setores industriais.

Entrevistas com representantes das empresas foram utilizadas para a coleta dos dados, gravadas para posteriormente serem transcritas (por volta de duas horas de duração por entrevista). Na parte inicial das entrevistas, os autores buscaram abordar de forma abrangente a caracterização dos programas Seis Sigma e Lean Production na companhia. Em um momento seguinte, abordaram os pontos principais dos programas, tais como seus benefícios, dificuldades, razões que levaram a implementação, etc. Quando necessário, os autores retomaram algumas perguntas do questionário que não haviam sido ainda respondidas pelos entrevistados. Ressalta-se, então, que as entrevistas não seguiram um roteiro fixo, caracterizando-se como entrevistas semi-estruturadas, utilizando-se um roteiro como direcionador para as entrevistas. Os entrevistados foram os responsáveis pelos respectivos programas: Black Belts nas empresas E1 e E3, e Master Black Belt na E2, responsável por ambos os programas e também gerente do escritório de projetos.

Posteriormente, foi feita uma análise da transcrição dos dados coletados nas entrevistas a fim de ser se observar as dificuldades da etapa de implementação e os resultados decorrentes da introdução das metodologias Seis Sigma e Lean Production. A análise dos dados utilizou-se de métodos e técnicas de análise de dados qualitativos em uma lógica geral indutiva, de acordo com Andrade (2002). 


\section{RESULTADOS DO TRABALHO DE CAMPO}

Nesta sessão são apresentados os resultados a partir dos dados coletados, num primeiro momento individualmente, junto a uma caracterização geral da empresa (dados adicionais em relação à Tabela 1); em seguida, é feita uma descrição do programa Seis Sigma e do Lean Production. Na sequência da descrição individual, é apresentada uma análise cruzada dos dados. Por razões de sigilo solicitado pelas empresas, seus nomes não são divulgados.

Tabela 1 - Caracterização das empresas estudadas.

\begin{tabular}{|c|l|l|l|l|}
\hline $\begin{array}{c}\text { Empr } \\
\text { esa }\end{array}$ & Setor Econômico & Faturamento [R\$] & Porte ( $\mathbf{n}^{\mathbf{0}}$ funcionários) & Origem \\
\hline E1 & Higiene e perfumaria & 3 bilhões & 3.000 & Nacional \\
\hline E2 & Automotivo & 600 milhões & 3.500 & Norte-americana \\
\hline E3 & Serviços de seguro & 1 bilhão & 1.800 & Espanhola \\
\hline
\end{tabular}

\section{Descrição da E1}

A E1 é uma empresa fundada em 1969 no setor de cosméticos e produtos de higiene e de perfumaria. Possui capital 100\% nacional e é uma das empresas líderes no mercado brasileiro. A empresa adota vendas diretas como seu modelo de vendas, possuindo consultoras ao invés de lojas (por volta de 400 mil consultoras). A empresa possui a certificação ISO 9001: 2000 e ISO 14001. Ademais, possui os programas de Gerenciamento por Processos, TPM (Total Productive Management) e CGMP (Current Good Manufacturing Practices).

A empresa implantou o programa Seis Sigma no ano em 2004 com o intuito de ampliar suas atividades no mercado internacional, especialmente o europeu. Durante a implantação do programa, a gerência responsável pela qualidade teve, no início, grande apoio da alta direção. O funcionário entrevistado destacou o apoio dos líderes da empresa como um fator essencial para se obter resultados eficazes com o programa. Cada funcionário, durante a implantação, recebia cerca de 20 horas de treinamento por ano. Os treinamentos foram realizados por meio de consultorias externas à organização e por funcionários "multiplicadores de conceito".

Uma vez que o programa apresentou problemas inesperados, a partir de 2005, o mesmo não teve mais prioridade dentro da organização. Após meses de 
implementação o apoio da alta gerência diminuiu drasticamente e constatou-se que o programa estava sendo utilizado para resolver problemas básicos do cotidiano. Assim, gerava-se, sem necessidade, um custo alto de investimento e de treinamento. Outro problema relatado diz respeito à utilização das ferramentas do Seis Sigma pelos funcionários encarregados da qualidade. O corpo de trabalhadores não via proveito em utilizar as ferramentas sofisticadas que aprendiam no treinamento e também não sabiam onde utilizá-las. Deste modo, os funcionários criaram certo receio pelo programa. Na E1 não havia diferenciação entre os Black Belts e Green Belts. Ambos eram vistos apenas como multiplicadores de conceito. Nesse mesmo sentido, para um Black Belts obter certificação, não era necessário finalizar um projeto, apenas ser treinado em ferramentas estatísticas.

De forma a eliminar, ou ao menos reduzir as barreiras encontradas, os gerentes optaram por fazer um replanejamento estratégico. O Seis Sigma foi interrompido e adotou-se o programa "Gestão de Processo". Os gerentes da empresa buscaram disseminar a ideia e aplicação das ferramentas básicas, sem, no entanto, apresentá-las como ferramentas Seis Sigma. Pretenderam assim, após capacitar o corpo de funcionários nas ferramentas do Seis Sigma, retomar aos poucos o programa, o que não foi identificado até a finalização desse estudo.

Em 2002 ocorreu o início da introdução do Lean Production. Os mesmos problemas apresentados durante a implantação do Seis Sigma ocorreram também com o Lean Production. A empresa não tinha condições básicas e não criou uma infraestrutura de funcionários para a sua implementação. Foram conduzidos alguns projetos de melhoria no escopo dos princípios do Lean Production, mas o programa não se estendeu para toda a empresa. Dessa forma, não houve a oportunidade de integração com o Seis Sigma.

\section{Descrição da E2}

A E2 é uma multinacional norte-americana que atua no ramo automotivo e possui participação expressiva no mercado mundial. A companhia possui como principais clientes as montadoras de automóveis, fabricantes de caminhões leves, médios e pesados, além dos fabricantes de maquinário e implementos agrícolas pesados. O grupo possui fábricas nos EUA, Inglaterra, Japão, China, México, Índia, 
Polônia e Brasil. Em relação à América Latina, a companhia concentra suas atividades no Brasil, onde possui fábricas principalmente no interior de São Paulo. A unidade visitada é responsável pela produção de transmissões e existe há mais de 40 anos.

O Seis Sigma foi implantado na unidade estudada no final da década de 90 com o treinamento dos primeiros Black Belts. No início foram 6 e, até a finalização do presente estudo, havia 5 Black Belts. Para atingir a certificação, um Black Belt deve finalizar dois projetos e fazer uma prova de conhecimentos sobre a metodologia. Pelo menos um dos projetos deve ter habilitação em estatística. Já os Green Belts têm como exigência para a sua certificação a conclusão de um projeto em um período de um ano.

A companhia divide os projetos em 3 níveis de uma pirâmide, como 0 entrevistado exemplificou. No topo da pirâmide estão os projetos mais complexos de longa duração e são os que provavelmente irão trazer maior retorno (aproximadamente 13 projetos por ano). Já no meio da pirâmide são os projetos de médio prazo que possuem duração de 3 a 4 meses (em torno de 80 projetos por ano). Por fim, no nível mais inferior situam-se os projetos que ocorrem com maior frequência por serem projetos mais simples que os demais (maioria dos projetos).

Por ser uma empresa característica de manufatura, grande parte dos projetos corresponde a projetos nas linhas de produção, mas também inclui projetos de desenvolvimento de novos produtos. Quando aplicado no desenvolvimento de produtos, a companhia utiliza o Design for Six Sigma (DFSS) que, segundo Harry e Schroeder (2000), é um método que permite desenhar um produto ou serviço desde o início do processo de desenvolvimento ou remodelar apenas parte deste. No entanto, esse método é internamente denominado Six Sigma for Design and Develop.

Um dos requisitos presentes nos projetos desenvolvidos diz respeito a relação com o planejamento estratégico. Os projetos devem estar alinhados com as estratégias e objetivos da organização a fim de promover e garantir sustentabilidade e crescimento. A companhia mede o retorno advindo de cada projeto por meio da medição do retorno financeiro, reduções de custos, reduções de inventário e satisfação do cliente externo. Essa última medição é realizada através de uma 
pesquisa de campo. A unidade visitada havia investido até a realização da entrevista a quantia aproximada de $\mathrm{R} \$ 225.000,00$ no programa e obteve cerca de o dobro desse valor em ganhos financeiros, conforme relatado pelo entrevistado.

Os princípios do Lean Production foram implantados na mesma época que se iniciou o programa Seis Sigma na companhia, ou seja, no final da década de 90, com o intuito de eliminar desperdícios através de trabalhos que não agregavam valor aos produtos produzidos. A unidade havia investido aproximadamente $R \$$ $375.000,00$ no programa e obteve mais de $R \$ 500.000,00$ de ganhos financeiros, de acordo com relato do entrevistado.

No início da implantação dos dois programas, havia uma concorrência por recursos (financeiros e de pessoal). Havia na unidade estudada uma auditoria interna para o programa Lean Production. Assim, quando se estava próximo da data para a realização da avaliação, toda a atenção e os recursos se voltavam para o Lean Production e, deste modo, os projetos do programa Seis Sigma eram postergados por um período.

A principal dificuldade encontrada para a introdução adequada do Lean Production diz respeito à cultura e postura dos funcionários perante a filosofia do programa. Conforme o entrevistado, muito mais que conhecer as ferramentas e suas aplicações, o funcionário deve primeiramente entender a filosofia por trás do programa, compreendê-la e considerá-la como parte da sua cultura. O gerente considera esse como um dos fatores-chave de sucesso para uma organização ter resultados eficazes.

A empresa alega que não há necessidade de iniciar a implantação de um programa para, posteriormente, implantar o outro. Também considera que os programas se completam: o Seis Sigma foca no resultado e na análise de dados, enquanto que o Lean Production possui uma forte filosofia de trabalho, característica que é escassa no Seis Sigma, de acordo com o entrevistado. A empresa vem utilizando o Seis Sigma para analisar os dados com todo enfoque estatístico que é característico do programa e utiliza o Lean Production como uma base de princípios e filosofia. Também acredita que um programa cobre os pontos fracos do outro, sendo que ambos atuam de forma complementar, como já destacado. 


\section{Descrição da E3}

Fundada em 1933, a empresa E3 atualmente opera em atividades de seguros, financeiras e de serviços. A empresa está presente em 38 países, possui cerca de 65 milhões de clientes. Atualmente, é o maior grupo segurador da Espanha. O grupo decidiu que a expansão da corporação para os países da América Latina era uma opção viável. Assim, em 1984 começaram as primeiras aquisições. Hoje a companhia está presente em 21 países da América e ocupa o primeiro lugar em volume de recursos e presença física na América Latina. O grupo iniciou suas atividades no Brasil em 1992, quando se deu a incorporação de um outro grupo segurador nacional. Nacionalmente, a empresa atua nos seguintes ramos do mercado de seguros: riscos pessoais e benefícios; garantia e seguros de crédito comercial; assessoria de resseguros; e seguros de pessoas e planos de previdência. Ao analisar de modo geral a estrutura organizacional da companhia, nota-se que é uma empresa integrada quanto à forma de gerir seus negócios, mas, ao mesmo tempo, é também uma companhia diversificada.

Em 2000, através de uma iniciativa do presidente da empresa, teve início a implantação do programa Seis Sigma, com o intuito de atender as exigências e expectativas dos clientes e manter a padronização dos processos para suportar o crescimento da organização. No organograma empresarial, a estrutura do Seis Sigma é separada das demais e esta alocada diretamente abaixo do vice-presidente da companhia.

O histórico do programa Seis Sigma na empresa pode ser divido em duas fases distintas. Na primeira fase a implantação do Seis Sigma se deu apenas em uma parte da empresa. O setor de seguros, que era o maior na época, não participava do programa. Já a segunda fase, envolve a disseminação do programa, a partir de 2003, na empresa com um todo. Como a decisão de implantação do programa veio de forma estratégica, direcionada pelo presidente, e por ser implantando em uma área reduzida da empresa, não se verificou resistência de aceitação do programa pelos diversos níveis hierárquicos da organização.

A implantação se deu através do treinamento da liderança com um grupo inicial de 4 Black Belts. Quando se realizou a entrevista, havia na companhia 8 Black Belts e 65 Green Belts. Havia também na empresa 17 projetos Seis Sigma em 
andamento, com previsão de chegar a aproximadamente 30 projetos por ano. A diferença básica entre os projetos dos Black Belts e dos Green Belts diz respeito ao escopo desses projetos. Os Green Belts realizam projetos em sua própria área de trabalho, mais focados e com escopo menor. Já os Black Belts, também pelo fato de estarem integralmente dedicados ao Seis Sigma, realizam projetos com escopo maior e que envolvem diversos setores da empresa.

Até o momento da realização da visita na empresa, foram investidos no programa aproximadamente $\mathrm{R} \$ 750.000,00$, obtendo-se um ganho financeiro com o programa mais de $\mathrm{R} \$ 2$ milhões. A maior dificuldade referente ao programa Seis Sigma ocorreu durante transição entre as duas fases já anteriormente citadas.

O Lean Production foi implantando na companhia juntamente com o Seis Sigma, também através de diretrizes do presidente da empresa. As razões que culminaram com a inserção do Lean Production foram as mesmas que as do Seis Sigma. Todos os projetos implantados devem estar estrategicamente alinhados com os objetivos organizacionais da empresa.

De acordo com os entrevistados (dois Black Belts e um Green Belt) há um vínculo forte entre os programas. Um dos entrevistados colocou que "é difícil ver o Lean Production sozinho, ele está diretamente ligado ao Seis Sigma. O Lean Production funciona como uma ferramenta do Seis Sigma". Com isso, na organização estudada, destaca-se a visão de que os programas são complementares.

Uma vez que há esse vínculo entres os programas, todos os funcionários que recebem treinamento referente ao Seis Sigma, também o recebem sobre o Lean Production. Os ganhos financeiros e o aumento da produtividade resultantes da implantação dos programas na empresa foram relatados como acima do esperado. Todavia, os entrevistados também relataram que a melhoria da qualidade dos serviços assim como a satisfação dos clientes seguiu conforme esperado, porém o market share cresceu de forma significativa e pode ser visto como resultado do sucesso dos programas. No entanto, não foram disponibilizados pela empresa valores numéricos referentes a estes resultados. Como não parece haver distinção significativa entre as atividades do Lean Production e do Seis Sigma na organização, 
os ganhos financeiros e os investimentos relatados sobre o segundo programa podem ser atribuídos de forma conjunta ao primeiro.

\subsection{Análise Cruzada dos Dados}

Este tópico tem como objetivo mostrar uma análise cruzada dos dados obtidos a partir dos dados coletados em campo. Conforme já apresentado anteriormente, as três empresas pertencem a setores industriais diferentes. Este fato possibilita uma melhor visualização sobre a integração e dificuldades decorrentes dos programas Seis Sigma e Lean Production em ramos industriais diferenciados. No entanto, não é pertinente uma generalização dos resultados para deferentes setores industriais. Outro aspecto importante é que as companhias são consideradas de grande porte e possuem um faturamento anual expressivo. Este aspecto favorece uma maior facilidade de investimento necessário para implantação e condução de programas de melhoria dessa natureza.

A Tabela 2 mostra uma síntese dos principais pontos de cada caso estudado. Como pode ser observado na tabela, a empresa E1 é a que apresenta a mais recente implementação do Seis Sigma. A E2 e a E3 concluíram mais de 25 projetos Seis Sigma por ano. Nas empresas E2 e E3, os investimentos feitos no Seis Sigma possuem cifras expressivas, assim como o retorno financeiro, o que demonstra resultados significativos decorrentes dos programas para as empresas. No entanto, os valores investidos e os ganhos financeiros com o programa não foram fornecidos pela E1, não permitindo uma constatação mais extensa em todas as organizações investigadas.

Todas as empresas possuem um número expressivo de Black Belts, e utilizam algumas das principais ferramentas estatísticas. Um resultado diferente do esperado é que a somente a E2 conta com um Master Black Belt.

Quanto a data de implementação, nota-se que duas das empresas analisadas adotaram o Lean Production há mais tempo, sugerindo um período suficiente para que o programa atingisse, nessas organizações um certo grau de maturidade. Já a última empresa teve a sua implementação mais tardia e também o programa interrompido, o que dificultou a análise sobre a sua maturidade. No entanto, devido 
ao curto tempo de duração do programa na empresa, é muito provável que este não tenha atingido qualquer nível de maturidade.

Na E2 e E3 constata-se que o programa Lean Production já apresenta certa maturidade, demonstrada pelo relativo grande número de projetos por ano e os expressivos retornos financeiros. Nota-se também uma relação favorável entre os valores investidos e esses ganhos financeiros, indicando um programa amadurecido. Finalmente, constata-se que os programas Seis Sigma e Lean Production são integrados somente nessas empresas (E2 e E3).

Tabela 2 - Síntese dos Dados das Empresas.

\begin{tabular}{|c|c|c|c|}
\hline & \multicolumn{3}{|c|}{ Casos } \\
\hline & E1 & E2 & E3 \\
\hline $\begin{array}{l}\text { Nível hierárquico da } \\
\text { área de Qualidade no } \\
\text { organograma }\end{array}$ & Responde ao $\mathrm{RH}$ & $\begin{array}{l}\text { Encontra-se sob a diretoria } \\
\text { de qualidade }\end{array}$ & $\begin{array}{l}2^{\circ} \text { nível hierárquico, respondendo } \\
\text { a Vice-Presidência }\end{array}$ \\
\hline $\begin{array}{l}\text { Data de implementação } \\
\text { do Seis Sigma }\end{array}$ & 2004 & 1999 & 2000 \\
\hline $\begin{array}{l}N^{\circ} \text { de projetos Seis } \\
\text { Sigma/ano }\end{array}$ & * & $\approx 30$ & $\approx 30$ \\
\hline $\begin{array}{l}\text { Investimento Seis Sigma } \\
{[\$]}\end{array}$ & * & $\approx \mathrm{R} \$ 175.000$ & $\approx 750$ mil desde 2003 \\
\hline Retorno Seis Sigma [\$] & * & $>\mathrm{R} \$ 500.000$ & > R\$ 2 milhões/ano \\
\hline $\begin{array}{l}\mathrm{N}^{\circ} \text { de pessoas treinadas } \\
\text { em Seis Sigma }\end{array}$ & * & * & $\approx 1650$ \\
\hline $\mathrm{N}^{\circ}$ Master Black Belts & 0 & 1 & 0 \\
\hline No Black Belts & 20 & 5 & 8 \\
\hline $\mathrm{N}^{\circ}$ Green Belts & * & 30 & 65 \\
\hline Ferramentas Utilizadas & $\begin{array}{l}\text { Diagrama de } \\
\text { Causa e Efeito, } \\
\text { Diagrama de } \\
\text { Pareto, Análise } \\
\text { de Variância, } \\
\text { FMEA, 5S, } 5 \\
\text { Porquês, PDCA, } \\
\text { Teste de } \\
\text { Hipótese, } \\
\text { Histograma }\end{array}$ & $\begin{array}{l}\text { FMEA, CEP, Diagrama de } \\
\text { Causa e Efeito, Histograma, } \\
\text { Diagrama de Pareto, } \\
\text { Diagrama de Dispersão, } \\
\text { Box Plot, Teste de } \\
\text { Hipóteses, Análise de } \\
\text { Variância, Testes não } \\
\text { Paramétricos, 5S, PDCA, } \\
\text { DMAIC, DFSS, DOE }\end{array}$ & $\begin{array}{l}\text { FMEA, CEP, QFD, Diagrama de } \\
\text { Causa e Efeito, Histograma, } \\
\text { Diagrama de Pareto, Diagrama } \\
\text { de Dispersão, Box Plot, Teste de } \\
\text { Hipóteses, Análise de Variância, } \\
\text { Testes não Paramétricos, } \\
\text { Círculos de Controle da } \\
\text { Qualidade, PDCA, DMAIC, } \\
\text { DFSS, DOE }\end{array}$ \\
\hline $\begin{array}{l}\text { Data de implementação } \\
\text { do Lean Production }\end{array}$ & $\begin{array}{l}2002 \text { (interrupção } \\
\text { em 2003) }\end{array}$ & 1999 & 2000 \\
\hline $\mathrm{N}^{0}$ de projetos Lean/ano & ** & $\approx 50$ & $\approx 30$ \\
\hline Investimento Lean [\$] & * & $\approx \mathrm{R} \$ 375.000$ & $\approx \mathrm{R} \$ 750.000$ \\
\hline $\begin{array}{l}\text { Retorno Lean } \\
\text { Production [\$] }\end{array}$ & * & $>\mathrm{R} \$ 500.000$ & > R\$ 2 milhões/ ano \\
\hline $\begin{array}{l}\text { Integração entre seis } \\
\text { sigma e Lean } \\
\text { Production }\end{array}$ & Inexistente & alta & alta \\
\hline
\end{tabular}




\section{CONCLUSÕES}

Primeiramente, pode-se considerar que os objetivos do presente trabalho ("demonstrar os resultados de um trabalho de campo, cujo propósito foi identificar os fatores importantes para a implementação Seis Sigma combinado com os princípios do Lean Production") foram alcançados. Com base nas empresas investigadas e na literatura, constata-se que os resultados obtidos no presente trabalho compõem um conjunto de evidências que auxilia a analisar e verificar a utilização do programa Seis Sigma combinado com os princípios do Lean Production.

Evidencia-se nos casos analisados que, ao iniciar os procedimentos de implementação dos programas Seis Sigma e Lean Production, é necessário primeiramente formar uma infraestrutura capaz de contribuir para o alcance dos objetivos da organização. Uma das maiores dificuldades apontadas pelos entrevistados engloba a formação dessa infra-estrutura. A disseminação da cultura e das técnicas do Seis Sigma, assim como do Lean Production, é um desafio que pode ser difícil de transpor, uma vez que depende fortemente dessa base estrutural. Observa-se também que, durante a fase de implementação dos programas, os recursos humanos foram decisivos para se obter resultados favoráveis. Em um dos casos mencionados, a falta de conhecimento técnico das ferramentas e da filosofia dos programas por parte dos funcionários, não trouxe os resultados esperados pela gerência e culminou com a interrupção do programa Lean Production. Tal paralisação resultou em desperdícios de recursos, sejam esses econômicos ou humanos. Conclui-se então que a infraestrutura deve ter, como um dos principais componentes, profissionais qualificados e treinados no sentido de formarem equipes responsáveis para a condução dos projetos.

Outro problema constatado diz respeito à competição, em um primeiro momento, de recursos entre os programas. Essa competição foi equacionada com o passar do tempo através da maturação dos programas, possibilitando, assim, em um compartilhamento de recursos entre os programas, o que culminou com resultados favoráveis. Foi possível observar que em duas das três empresas estudadas havia uma forte interação entre o Seis Sigma e o Lean Production. Em uma das organizações, os Black Belts consideram os dois programas como apenas um, colocando os princípios do Lean Production como ferramentas do Seis Sigma. Na 
terceira empresa, não foi possível analisar a interação entre os dois programas, pois o Lean Production foi interrompido há alguns anos. Esta interação favorável entre os programas trouxe benefícios para as empresas, o que pode ser verificado por meio do expressivo retorno financeiro.

O presente trabalho apresenta, no entanto, limitações. Alguns dados e informações requeridas não puderam ser fornecidos pelas empresas visitadas por questões de sigilo. Apesar de esse problema, os resultados da demonstração realizada podem ser considerados como positivos, atendendo aos objetivos propostos. Deve-se também levar em conta que as conclusões deste estudo estão condicionadas ao tamanho limitado da amostra analisada. Apesar de se tratar de casos múltiplos, não permite uma generalização dos resultados, seja em função dos temas ou dos setores industriais dos quais as empresas pertencem. Porém, é possível um certo nível de generalização analítica, mas de forma limitada. Assim, pretende-se estender o trabalho de campo a fim de se obter uma maior abrangência para confirmar os resultados do presente trabalho, principalmente em empresas que alegam utilizar os programas em conjunto, ou seja, o Lean Six Sigma.

\section{Agradecimentos}

Os autores agradecem ao CNPq pelo apoio a condução do trabalho (Edital Universal) e concessão de bolsas via Programa PIBIC-CNPq da USP. Agradecem também ao Rogério Confortini que trabalhou na parte inicial do projeto de pesquisa, bem como as empresas que possibilitaram a coleta de dados. No entanto, cabe destacar que o trabalho reflete a visão dos autores e não das empresas pesquisadas. Finalmente, agradecem a contribuição dos revisores da Produção OnLine pelas sugestões e recomendações que significativamente contribuíram para a melhoria do artigo.

\section{REFERÊNCIAS}

ACHANGA, P.; SHEHAB, E.; ROY, R.; NELDER, G. Critical success factors for lean implementation within SMEs. Journal of Manufacturing Technology Management, v.17, n.4, p. 460-71, 2006. 
ANDERSON, R.; ERIKSSON, H.; TORSTENSSON, H. Similarities and differences between TQM, six sigma and lean. The TQM Magazine, v.18, n.3, p. 282-96, 2006.

ANDRIETTA, J. M.; CAUCHICK MIGUEL, P. A. Aplicação do programa seis sigma no Brasil: resultados de um levantamento tipo survey exploratório-descritivo e perspectivas para pesquisas futuras. Gestão \& Produção, v. 14, n. 2, 2007.

ANNAND, R.B.; SHUKLA, S. K.; GHORPADE, A.; TIWARI, M. K.; SHANKAR, R. Six sigma-based approach to optimize deep drawing operation variables. International Journal of Production Research, v.45, n.10, p. 2365-85, 2007.

ANTONY, J.; BANUELAS, R. Critical success factors for the successful implementation of six sigma projects. The TQM Magazine, v.14 n.2, p. 92-9, 2002.

ANTONY, J.; BANUELAS, R. Key Ingredients for the effective implementation of six sigma program. Journal of Measuring Business Excellence, v. 6, n.4, p. 20-7, 2002.

ANTONY, J. Some pros and cons of six sigma: an academic perspective. The TQM Magazine, v.16, n.4, p. 303-6, 2004.

ANTONY, J. Six Sigma for service processes. Business Process Management Journal, v.12, n.2, p. 234-48, 2006.

ANTONY, J. Some perspectives from leading academics and practitioners. International Journal of Productivity and Performance Management, v. 60, n. 2, 2010.

ARNHEITER E.D.; MALEYEFF, J. The integration of lean management and six sigma. The TQM Magazine, v.17, n.1, p 5-18, 2005.

ARTHUR, J. Lean six sigma demystified. United States of America: Mc Graw-Hill, 2007.

BASU, R. Six-sigma to operational excellence: role of tools and techniques. International Journal of Six Sigma and Competitive Advantage, v.1, n.1, p. 4464, 2004

BENDELL, T. A Review and comparison of six sigma and the lean organizations. The TQM Magazine, v. 18 n. 3, p. 255-62, 2006.

BHUIYAN, N.; BAGHEL, A. An overview of continuous improvement: from the past to the present. Management Decision, v. 43 n.5, p.761-71, 2005.

BHUIYAM, N.; BAGHEL, A.; WILSON, J. A Sustainable continuous improvement methodology at an aerospace company. International Journal of Productivity and Performance Management, v. 55, n. 8, p. 671-87, 2006. 
BOSSERT, J. Lean and six sigma: synergy made in heaven. Quality Progress, v.36, n. 7, p. 31-32, 2003.

BROTHERTON, B.; SHAW, J. Towards an identification and classification of critical success factors in uk hotels plc. International Journal of Hospitality Management, v.15, n.2, p. 113-35, 1996.

BYRN, G.; LUBOWE, D.; BLITZ, A. Using a lean six sigma approach to drive innovation. Strategy \& Leadership, v.35, n.2, p. 5-10, 2007.

CAUCHICK MIGUEL, P.A. Estudo de caso na engenharia de produção: estruturação e recomendações para sua condução. Produção, v. 17, n. 1, p.216-229, 2007.

CHAPMAN, R.L.; HYLAND, P.W. Continuous improvement strategies across selected Australian manufacturing sectors. Benchmarking for Quality Management \& Technology, v.4, n.3, p. 175-188, 1997.

CHIARINI, A. Japanese total quality control, TQM, deming's system of profound knowledge, BPR, lean and six sigma: comparison and discussion. International Journal of Lean Six Sigma, v. 2, n. 4, p. 332-355, 2011.

CLETO, M. G.; QUINTEIRO, L. Gestão de projetos através do dmaic: um estudo de caso na indústria automotiva. Revista Produção Online, v.11, n.1, p.210-239, 2011.

CUDNEY, E.; ELROD, C. A comparative analysis of integrating lean concepts into supply chain management in manufacturing and service industries. International Journal of Lean Six Sigma, v.2, n.1, p. 5-22, 2011.

DEVANE, T. Integrating lean six sigma and high performance organizations. San Francisco: Pfeiffer Wiley Imprint, 2004.

FERNANDES, M.M.; TURIONI, J.B. Seleção de projetos seis sigma: aplicação em uma indústria do setor automobilístico. Produção, v. 17, n. 3, p. 579-591, 2007.

FERNG, J.; PRICE, A.D.F. An Exploration of the synergies between six sigma, tqm, lean construction and sustainable construction. International Journal of Six Sigma and Competitive Advantage, v.1 n.2, p. 167-87, 2005.

FIORE, C. Lean Strategies for product development: achieving breakthrough performance in bringing products to market. Wisconsin: Quality Press, 2003.

GEORGE, M.L. Lean six sigma, combining six sigma quality with lean speed. New York: McGraw-Hill, 2002.

HARRY, M.; SCHROEDER, R. Six sigma: a breakthrough strategy for profitability. New York: Ed. Doubleday, 2000. 
HINES, P.; HOLWEG, M.; RICH, N. Learning to evolve: a review of contemporary lean thinking. International Journal of Operations \& Production Management, v.24, n.10, p. 994-1011. 2004.

HUANG, C. C.; LIU, S. H. A novel approach to lean control for taiwan-funded enterprises in mainland china. International Journal of Production Research, v.43, n.12, p. 2553-75, 2005.

JEYARAMAN, K.; TEO, L. K. A Conceptual framework for critical success factors of lean six sigma: implementation on the performance of electronic manufacturing service industry. International Journal of Lean Six Sigma, v.1, n.3, p. 191-215, 2010.

KUMAR, M.; ANTONY, J.; SINGHC, R.K.; TWIARID, M.K.; PERRY, D. Implementing the lean sigma framework in an indian sme: a case study. Production Planning \& Control, v.17, n.4, p.407-23, 2006.

MADER, D. P. Lean Six Sigma's evolution: integrated method uses different deployment models. Quality Progress, p. 40-8, 2008.

MARTIN, J. W. Lean six sigma for supply chain management, the 10-step solution process. New York: McGraw-Hill, 2007.

MCADAM, R.; DONEGAN, S. A comparative analysis of trilateral and concurrent business improvement methodologies in the high technology sector. International Journal of Manufacturing Technology and Management, v. 5 n.3, p.210-31, 2003.

MENDOZA, G. Competitive manufacturing. Proceedings of the $8^{\text {th }}$ Annual IQPC Six Sigma Summit, Miami, FL, 2007.

MONTGOMERY, D. C. A modern framework for achieving enterprise excellence. International Journal of Lean Six Sigma, v.1, n.1, p.56-65, 2010.

MOSCONE, D. Lean six sigma at GE. Proceedings of the $8^{\text {th }}$ Annual IQPC Six Sigma Summit. Miami, FL, 2007.

NASLUND, Dag. Lean, six sigma and lean sigma: fads or real process improvement methods? Business Process Management Journal, v. 14, n. 3, 2008.

PEPPER, M.P.J.; SPEDDING, T.A. The evolution of lean six sigma. International Journal of Quality \& Reliability Management, v.27, n.2, p. 138-155, 2010.

PIL, F.K.; FUJIMOTO, T. Lean and reflective production: the dynamic nature of production models. International Journal of Production Research, v.45 n.16, p. 3741-61, 2007.

PORTER, L. Six sigma excellence. Quality World, 2002. Disponível em: http://www.iqa.org. Acesso: 22/06/2006. 
PROUDLOVE, N.; MOXHAM, C.; BOADEN, R. Lessons for lean in healthcare from using six sigma in the nhs. Public Money \& Management, v.28, n.1, p. 27-34, 2008.

RAISINGHANI, M.S.; ETTE, H., PIERCE, R., CANNON, G.; DARIPALY, P. Six sigma: concepts, tools, and applications. Industrial Management \& Data Systems, v.105, n.4, p. 491-505, 2005.

SALAH, S.; RAHIM, A.; CARRETERO, J.A. The Integration of six sigma and lean management. International Journal of Lean Six Sigma, v.1, n.3, p. 249-274, 2010.

SCHROEDER, R.G.; LINDERMAN, K.; LIEDTKE, C.; CHOO, A.S. Six sigma: definition and underlying theory. Journal of Operations Management, v.26, n.4, p. 536-54, 2008.

SHEEHY, P.; NAVARRO, D.; SILVERS, R.; KEYES, V.; DIXON, D. The black belt memory jogger. Salen, OR: GOAL/QPC and Six Sigma Academy, 2002.

SHERIDAN, J. H. Lean sigma synergy. Industry Week, v. 249, n.17, p. 81-2, 2000.

SMITH, B. Lean and six sigma: a one-two punch. Quality Progress, v. 36 n. 4, p. 3741, 2003.

SNEE, R.D. Six-sigma: The Evolution of a 100 Years of Business Improvement Methodology. International Journal of Six Sigma and Competitive Advantage, v.1, n. 1, p. 4-20, 2004.

SNEE, R.D. When worlds collide: lean and six sigma. Quality Progress, p. 63-5, set. 2005.

SNEE, R.D. Lean six sigma: getting better all the time. International Journal of Lean Six Sigma, v.1, n.1, p. 9-29, 2010.

SNEE, R.D.; HOERL, R.W. Integrating lean and six sigma: a holistic approach. Six Sigma Forum Magazine, v. 6, n.3, p. 15-21, 2007.

STRAATMANN, J. Estudo das práticas adotadas por empresas que utilizam a produção enxuta em paralelo ao seis sigma no processo de melhoria. São Carlos: USP/SC, 2006. Dissertação de Mestrado em Engenharia de Produção, da Escola de Engenharia da Universidade de São Paulo (São Carlos), 2006.

WEDGWOOD, I. Lean sigma, a practitioner's guide. Upper Saddle River, N.J.: Prentice-Hall, 2006.

WELCH, J. Six Sigma leaders. Quality, v. 44, n. 3, p. 80, 2005.

WOMACK, J.P; JONES, D.T. A mentalidade enxuta nas empresas: elimine o desperdício e crie riqueza. Rio de Janeiro: Campus, 2004. 
YANG, C.C. An integrated model of tqm and ge-six sigma. International Journal of Six Sigma and Competitive Advantage, v.1, n.1, p. 97-111, 2004.

YUSUF, Y.Y.; ADEYELE, E.O. A comparative study of lean and agile manufacturing with a related survey of current practices in the UK. International Journal of Production Research, v.40 n.17, p.4545-62, 2002.

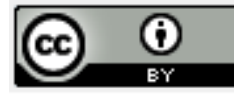

Artigo recebido em 12/05/2012 e aceito para publicação em 26/04/2013. 